\title{
The effects of hydration on the collagen and gelatine phases within parchment artefacts
}

\author{
Lee Grant Gonzalez ${ }^{*}$ and Timothy James Wess
}

\begin{abstract}
This review discusses the need to understand the effects of relative humidity on parchment and the current understanding of parchment structure and hydration. It also provides a critical evaluation of the body of foregoing research investigating the effects of relative humidity on parchment artefacts. The critical evaluation shows that the current scientific evidence that can be used to inform the debate with regard to relative humidity guidelines for the preservation of parchment artefacts is insufficient, especially in the light of a greater understanding of parchment based documents when considered as composite materials comprising collagen and gelatine. The differential behaviour of collagen and gelatine (and consequently the effect on the interfaces between them) in terms of response to relative humidity changes, is proposed as a key factor to be considered for future studies. This review concludes with an analysis of the next steps required in parchment research in order to provide an informed advisory framework.
\end{abstract}

Keywords: Parchment, Relative humidity, Collagen, Gelatine

\section{Introduction}

Parchment artefacts are composed of collagen, its degraded form gelatine, varying minerals, lipids, and for documents inks and paints. The main structural components are collagen and gelatine and are both hygroscopic. Changes to the relative proportions of collagen and gelatine (that may be mediated through adverse storage conditions and accelerated by the effects of lipids, or inks) contribute to the dominant effect of the mechanical properties of a parchment. It has also been shown that the high quantities of gelatine are related to physical features of parchment that may be undesirable, such as cockling [1]. Understanding the relationship between the mechanical and thermodynamic properties of parchment structure and water content is therefore critical to establishing appropriate temperature (Ta) and relative humidity $(\mathrm{RH})$ conditions for the storage, display, and preservation of parchment artefacts. When establishing these parameters, it is important for the structural organisation of collagen and gelatine to be considered because water interaction with collagen is found to be different to gelatine (discussed later).

\footnotetext{
* Correspondence: GonzalezLG@cf.ac.uk

School of Optometry and Vision Sciences, Cardiff University, Maindy Road, Cardiff CF24 4 LU, UK
}

Parchment, collagen and gelatine structure and hydration Work in the last 10 years using techniques such as Microfocus X-ray diffraction have shown that within parchment, the collagen and gelatine components are found in discreet layers where the majority of gelatine is localised on the surface [2]. This finding has been supported through the use of Attenuated Total Reflection Fourier Transform Infrared Spectroscopy (ATR-FTIR) that recorded a spectrum characteristic of gelatine at the surface of parchment samples and when those surfaces were scraped and a repeat measurement performed a spectrum characteristic of collagen was recorded [3]. Parchment artefacts can therefore be described structurally, as a matrix of collagen fibres interfaced with a gelatine exterior. This structural layering is due to both collagen denaturation caused by the highly alkali liming step (calcium hydroxide) during the manufacturing process and the exposure of the surface to other agents that cause denaturation- post production- such as UV light [4]. As well as the distribution described of collagen and gelatine through parchment, there are also specific loci of gelatine that seems to relate to the presence of hair holes in samples [5], this may be due to elevated levels of endogenous lipid in these areas that seems to correlate with collagen degradation [6]. Other agents 
such as metal based inks also lead to localised collagen degradation around inked areas throughout the depth of the parchment sheets [7].

The structure and hierarchical organisation of collagen is well documented. The process of constructing a collagen tissue begins with three collagen polypeptide chains each comprising over 1050 amino acids of a specific sequence twisting to form a collagen molecule with a rigid rope like triple helix motif. The alternate direction of the twist and the steric local arrangement of amino acids in the chain cause the helix to lock into a specific structure where water has a significant relationship with the collagen molecule. Collagen molecules then aggregate in a very specific and highly directed way where stability is engendered through ionic interactions, hydrophobic regions and covalent intra and intermolecular crosslinkages to form an insoluble collagen fibril. Collagen fibrils are slender structures $(\approx 120 \mathrm{~nm}$ wide in skin and at least several microns long) each fibril comprises several thousand collagen molecules in cross-section and extends over several collagen molecules in length. Fibrils are organised less specifically into bundles to form a collagen fibre, and finally collagen fibres form a collagen tissue [8].

The stability of the collagen triple helix is in part due to the relationship of collagen with water at specific levels of the hierarchical organisation within a tissue. Water molecules can be classified as associating with collagen in a tissue in four different phases: structural water (phase I) bound tightly within the collagen triple helix; either through a double or triple hydrogen bond. Bound water (phase II), where water molecules form bridges between neighbouring polypeptide chains and act as receptors for $\mathrm{CH}-\mathrm{O}$ hydrogen bonds [9]. Free water (phase III) where water is fixed by one hydrogen bond between polypeptide chains or fixed in the hole zones at the end of the polypeptide chain, and finally transition water or unperturbed bulk water (phase IV), where water molecules are bound between the fibrils $[10,11]$. The total amount of structural and bound water (phase I and II) contained in collagen is approximately $0.5 \mathrm{~g} / \mathrm{g}$ [12-14]. The remaining amount of free and bulk water (phase III and IV) held in a collagen tissue is dependent upon RH.

In its purest sense, gelatine can be defined as a polypeptide chain in a random coil configuration. Such a structure can be derived from the denaturation and unfolding of collagen triple helices, this form has a greater affinity to water than collagen, and gelatine is therefore characterised as a soluble protein. In some instances, depending upon the denaturation mechanism, the native polypeptide chains may have fragmented into smaller amino acid sequences. Therefore to characterise different gelatine structures, gelatine can also be defined as having a molecular weight distribution of 10- 300Daltons. The process of collagen denaturation involves two agents. Firstly an agent to perturb the collagen triple helix motif and its bonding relationship with other collagen molecules, such as low or high $\mathrm{pH}$, UV irradiation or heat [15]. Secondly the presence of water is needed to allow the space for the collagen helix to unwind and for the hydrogen bonding to be redistributed [16]. A point of debate is the likelihood that agents such as UV irradiation or pollution can impose effects of bond scission in low levels of hydration, but do not allow sufficient space for the collagen helix to unfold. It may therefore be possible that the collagen remains in a pre-gelatinised state that is difficult to distinguish from native collagen without further perturbing the system by attempting to extract the collagen and examine its physical properties or integrity. In comparison to collagen, gelatine is an entropically more stable state and does not exhibit any recognisable regular structures. When collagen triple helices unfold the structural integrity of the collagen fibril and fibres are lost. The fibre bundles are observed to become thinner and start to lose cohesion. Small open spaces will appear between the bundles, which are interspersed with crisscrossed, tangled, thin collagen fibres [17]. Collagen fibres bundles will start to lose orientation and split apart causing fibres to form a gel and the collagen tissue concomitantly losing its mechanical strength [18].

In a gelatine structure, water mediated hydrogen bonds will form with any available polar amino acid side chain [19]. These are: the charged groups of the amino acid side chains such as lysine, arginine, glutamic and aspartic acids, hydroxyl groups of the side-chains in serine, threonine and hydroxylysine; and the carbonyl groups and the $\mathrm{N}-\mathrm{H}$ groups of side chains and main chain polymers not involved in intramolecular bonding [20]. In the unfolded collagen structure, more polar groups are exposed to water, gelatine can therefore bind to significantly more water molecules than the original collagen structure. Yakimets et al. [21] studied how water interacts with gelatine and reported that for gelatine type B with a high bloom index (225) a complete monolayer of water on the structure was achieved at $14 \%$ water content. Between 14-22\% water content, multilayers of less strongly interacting water are formed, and above $22 \%$ the water appears to behave as an unperturbed bulk phase.

In Table 1 the percentage water contained in purified Type I collagen and gelatine at different $\mathrm{RH}$ and $\mathrm{Ta}$ is given. It is generally observed that in protein structures such as collagen, the absorption of water increases as a sigmoidal function of the $\mathrm{RH}$ [22] this typically indicates saturation phenomena and indicates that different levels of water-collagen interactions may occur. It can also been seen that the amount of water held in the collagen at a specific $\mathrm{RH}$, is lower as the temperature increases from 25 to $40^{\circ} \mathrm{C}$, which shows the intimate relationship 
Table 1 The percentage water content in collagen and gelatine at varying $\mathrm{RH}$

\begin{tabular}{ccccc}
\hline & Collagen & \multicolumn{3}{c}{ Gelatine } \\
$\mathbf{T m}{ }^{\circ} \mathbf{C}$ & $\mathbf{2 5}$ & $\mathbf{4 0}$ & $\mathbf{2 5}$ & $\mathbf{4 0}$ \\
\hline$\% \mathrm{RH}$ & & & & \\
5 & 5.45 & 4.18 & 5.30 & 3.99 \\
10 & 7.39 & 6.33 & 6.75 & 5.95 \\
20 & 10.06 & 8.89 & 8.71 & 8.40 \\
30 & 12.44 & 11.64 & 11.25 & 10.89 \\
40 & 15.14 & 14.46 & 13.82 & 13.34 \\
50 & 17.95 & 17.23 & 16.30 & 15.70 \\
60 & 21.06 & 20.16 & 18.48 & 17.64 \\
70 & 25.05 & 23.78 & 21.60 & 20.05 \\
80 & 30.20 & 28.71 & 26.73 & 25.35 \\
90 & 40.70 & 37.35 & 40.20 & 36.45 \\
95 & 50.01 & 44.75 & 57.60 & 49.05 \\
\hline
\end{tabular}

Percentage of water absorbed by dry protein as a function of the $\mathrm{RH}$ at $25^{\circ} \mathrm{C}$ and $40^{\circ} \mathrm{C}$ as determined by [23]. Increases in temperature will lower the percentage water in the collagen and gelatine in the order of $0.5-2 \%$ depending upon the $\mathrm{RH}$.

between RH, Ta and the water content of the sample. In comparison, gelatine holds less water than collagen at the same $\mathrm{RH}$ and $\mathrm{Ta}$ until $95 \% \mathrm{RH}\left(25^{\circ} \mathrm{C}\right)$ when gelatine holds $7.6 \%$ more water than collagen. Moreover, in the gelatine structure, the characteristic sigmoidal change in water observed with increasing $\mathrm{RH}$ is no longer found. The amount of water held in the gelatine is seen to increase in increments of $\approx 2-2.5 \%$ for the $\mathrm{RH}$ range of $10-60 \% \mathrm{RH}$ after which the amount of water increases exponentially until the gelatine dissolves. It is interesting from the data presented in Table 1 that collagen and gelatine hold different percentages of water at the same $\mathrm{RH}$, because this implies that in composite collagen and gelatine materials, such as parchment, the percentage of overall water content in the collagen and gelatine phases will be different.

We postulate that the differences in water content between the collagen gelatine phases within parchment is a critical link when determining appropriate $\mathrm{RH}$ conditions for the storage, handling and display of parchment. The specific interaction between water and collagen at different levels of molecular and supramolecular structure means that changes in water content will induce well described and characteristic structural changes at each level; however in gelatine, the lack of conformational specificity means that changes in water content can only be described by the relative level in the proximity and entanglement of the collagen polypeptide chains. The gelatine-collagen stratigraphy within parchment may mean that the difference in water content at any given $\mathrm{RH}$ could cause differences in the cohesion between these structural phases $[24,25]$. It is also possible that the difference in structural stability between the collagen and gelatine phases could affect mechanical properties within parchment inducing a stress at the interfaces between collagen and gelatine. If the changes in water exposure are cyclic, the stress imposed could eventually lead to mechanical fatigue and fracture, this would be a feed forward mechanism in which collagen is further damaged and converts to gelatine. Because the collagen, gelatine and interface layers will absorb different amounts of water at the same $\mathrm{RH}$, there will be a differential distribution of water at any given RH typically used in storage conditions; an alteration in the RH will cause the water distribution and the physical properties of the different components to change [26]. The effect that the disparity between water content of collagen and gelatine has on parchment stability is not clear, and needs to be resolved. Electron micrograph images of parchment surfaces show a delamination of a glassy surface layer of parchment that has separated in parts from the underlying core of the parchment. These fractures are possible representative of differential behaviour of layers and interfaces within the parchment document [27].

The preceding section describing parchment and its structural components collagen and gelatine has shown that the structural behaviour of parchment artefacts with changing water content will vary depending on the relative proportions of collagen and gelatine in the parchment object. This has implications for setting environmental standards for the preservation of parchment artefacts because it means that not all parchment artefacts will react in a similar way, at the same RH. Aliev [28] showed that the water content differed between 20th century (new) and 17th -19 th century (historical) parchments by approximately $3 \%$ and that a proportion of water was held in parchment by the lipid component. The water content of 20th century (new), 17th century book (historical) binding and 14th century (medieval) parchments at different $\mathrm{RH}$ has been reported by [29], these are given in Table 2 . Compared to the new parchment, the observation was

Table 2 Percentage water content in parchment at varying $\mathbf{R H}$

\begin{tabular}{cccc}
\hline Tm ${ }^{\circ} \mathbf{C}$ & New & Historical & Medieval \\
\hline$\% \mathrm{RH}$ & $\mathbf{4}$ & $\mathbf{4}$ & $\mathbf{4}$ \\
55 & $14(+/-1.0)$ & $11(+/-0.8)$ & $16(+/-1.1)$ \\
65 & $19(+/-1.3)$ & $18(+/-1.3)$ & $21(+/-1.5)$ \\
75 & $21(+/-1.5)$ & $20(+/-1.4)$ & $24(+/-1.7)$ \\
86 & $23(+/-1.6)$ & $20(+/-1.4)$ & $25(+/-1.8)$ \\
95 & $26(+/-1.8)$ & $24(+/-1.7)$ & $28(+/-2.0)$ \\
98 & $26(+/-1.8)$ & $23(+/-1.6)$ & $29(+/-2.1)$ \\
\hline
\end{tabular}

Percentage of water absorbed by parchments as a function of the $\mathrm{RH}$ at $4^{\circ} \mathrm{C}$ as determined using equilibrating weights by [29]. 
that historical parchment samples absorb less water over the $\mathrm{RH}$ range investigated, and medieval parchment samples absorbed more water. However, it remains unclear from either of these studies how much water was held in each of the collagen or gelatine phases of the parchment, this was due to the limitations of the analytical technique used.

\section{A critical assessment of research investigating changes of parchment artefacts with alteration of $\mathrm{RH}$}

Ideally, the RH range for parchment storage, display and handling would minimise a) the conversion of perturbed/ pre-gelatinised collagen into gelatine, b) reduce the loss of water from the parchment, which will increase the brittleness, c) reduce mould growth and d) be cost effective. Therefore the key to determining the most appropriate $\mathrm{RH}$ for parchment storage, display and handling is to understand the range of $\mathrm{RH}$ that parchment can be exposed to before there is sufficient space between neighbouring collagen fibrils for them to unfold, and determine the relationship between structural changes and water content. Because parchments are composite materials understanding how water content affects the thermodynamic stability and the mechanical properties is challenging. At present, the recommended standards for storing parchment are based upon recommendations for water absorbent materials (hygroscopic). For European climates the recommended $\mathrm{RH}$ is $55 \%$ [30], the midpoint between the upper limit of $70 \%$, where the environment is favourable for mould germination [31] and $40 \%$ the point at which hygroscopic materials (in general) can become brittle.

Table 3 shows a summary of recommended RH conditions for the storage of parchment from several studies. In the study by Weiner et al. [32] the relative proportions of collagen and gelatine in Dead Sea Scroll (DSS) samples were measured, and it was recommended that the upper $\mathrm{RH}$ limit for parchment storage should not exceed $65 \%$. This value is based on their reasoning that the amount of water needed to cause the remaining collagen component of the scrolls to denature: 'Optimal preservation conditions, which minimise the rate of transformation (of collagen into gelatine) should be established'. Our reasoning agrees with that of Weiner's; however, Weiner's study does not furnish the reader with the evidence to account for a storage upper limit of $65 \%$

Table 3 Summary of the RH range recommended in the literature

\begin{tabular}{lcc}
\hline Authors & \multicolumn{2}{c}{ Recommended RH } \\
\cline { 2 - 3 } & Low & High \\
\hline Weiner, et al., [32] & - & $65 \%$ \\
Schilling, et al, [34] & $35 \%$ & $40 \%$ \\
Hansen, et al., [37] & $30 \%$ & $40 \%$ \\
\hline
\end{tabular}

$\mathrm{RH}$. The figure of $65 \% \mathrm{RH}$ is based a upon the absorption of water in rat tail tendon (RTT) collagen and is cited from the work by Gustavson [33]. Here, Gustavson showed that water intake for dry rat tail tendon collagen is linear up to an $\mathrm{RH}$ of $65 \%$, after which the amount of water absorbed by collagen is shown to increase exponentially with water vapour pressure. Firstly, Gustavson's work does not provide any evidence to suggest that an $\mathrm{RH}$ above $65 \%$ will increase the transformation of collagen into gelatine; since hydrating collagen does not cause denaturation unless the collagen integrity has been compromised in some way. Although it may be appropriate to model the hydration changes of collagen in parchment with those of tendon; given that they are both made of predominantly Type I collagen fibrils, it is not optimal to do so for a whole parchment artefact. This is because extracted tendon, in comparison to parchment, is at least $95 \%$ collagen $\mathrm{W} / \mathrm{W}$ with minimal gelatine content and some non-collagenous components such as proteoglycans and elastic proteins.

The DSS study by Schilling et al. [34] measured the change in volume of parchment from water absorption and desorption (the hygric expansion coefficient) at different RH. This study found that the rate of water absorption was slowest and the volume change lowest in the degraded new (control) parchment samples. They also found that the rate of absorption and volume change were in the same order of magnitude in the DSS samples as gelatine samples used. Specifically, the data showed that at an $\mathrm{RH}$ of $38-46 \%$ the hygric expansion coefficient was lowest for almost all samples including the control samples of new parchment and new gelatine (obtained from Kremer Pigmente, biological source unknown). This indicates that in this $\mathrm{RH}$ range the parchment volume change was lowest, suggesting that equilibrium was reached. The study also found, using dynamic mechanical analysis (DMA), that the internal stresses (resistance of the material to external forces) on new parchment were considerably larger when samples were exposed to conditions below $30 \% \mathrm{RH}$. This indicates that 30\% RH is a critical point where parchment loses sufficient water to cause a change in the mechanical properties of parchment.

The study by Dernovšková, et al.,[35] investigated the amount of water absorbed by parchments at different $\mathrm{RH}$, and found that 16th century (historical) parchments typically absorb more water than new parchments, and that the maximum water absorption was achieved at $45.5 \% \mathrm{RH}$ and $28.1 \% \mathrm{RH}$ respectively. Although no evidence was provided in the study, it is possible to speculate that the difference in the amount of water absorbed and the $\mathrm{RH}$ when maximum water absorption was reached were the result of a difference in the proportions of collagen and gelatine between samples. Older parchments are often found to have larger proportions 
of gelatine because they have usually been exposed to damaging environmental conditions, such as UV light, for much longer.

These studies usefully show that there are changes in the physical properties of parchment artefacts at different $\mathrm{RH}$ and this may be caused by the proportion of collagen and gelatine present in the parchment. Unfortunately neither Schillings nor Dernovšková's study used a large enough sample set to provide sufficient data for the broad spectrum of parchment artefacts. Schilling only used DSS samples, which validates the results for a very specific sample set, and Dernovškovás study only compared goat parchments from 1550 AD. Therefore, these sample sets need to be augmented by further studies to generalise the case and be representative of the range of parchment artefacts in terms of manufacturing procedures, animal skins used or the type of post manufacture storage conditions. In addition, techniques need to be developed to estimate the amount of collagen and gelatine in each sample and relate this to the physical changes observed at different $\mathrm{RH}$ values, although the properties of the structural components of parchment (gelatine and collagen) have been characterised in part by response to $\mathrm{RH}$, there is a need to consider the spatial relationship of collagen and gelatine at a variety of length scales within each parchment.

The study by Bowden, et al. [36] further demonstrated there was a difference between new and historic parchments by measuring the thermal response (the temperature change from exothermic or endothermic reactions induced by hygrometric changes i.e. gain or loss of unbound water) of parchments during $\mathrm{RH}$ changes. The study found that the thermal peak area, which represents the temperature change of parchment when exposed to different $\mathrm{RH}$, was related to the mass of the sample. The main finding provides evidence that the hygrometric change (i.e. water exchange) in gelatine and the historical parchment samples (15th-18th century) is much slower than in new parchment samples, thus indicating that the behaviour of parchment artefacts at varying $\mathrm{RH}$ relates to the proportions of collagen and gelatine they contain. The results of this study show the thermal response method is suitable to demonstrate the response of parchment to $\mathrm{RH}$ change; however, it did not provide any specific guidance for setting environmental parameters for parchment.

Hansen et al. [37] is the key study that recommends $\mathrm{RH}$ conditions based upon the mechanical changes of parchment that occur as a function of RH. Hansen measured the load to break, energy to break, and percentage strain to break, with the aim of quantifying the amount of force needed to induce mechanical failure and showed how this changes with the amount of water held in a parchment. Hansen also measured the initial modulus and restrained force, with the aim of quantifying the resistance of parchment to structural alteration with respect to $\mathrm{RH}$ and therefore a change in the water content.

The initial modulus experiments made by Hansen is a measure of the parchment elasticity and are a direct measure of how the increase or decrease in water mediated bonds contributes to a change in the brittleness of the samples. This gives an understanding of which $\mathrm{RH}$ may alter a parchments physical properties and ability to resist structural deformations. Because of the high coefficient of variance in the tensile measurement (as high as $32-34 \%$ in some cases), the study did not show a significant difference in the initial modulus of the parchments at any RH except $11 \%$ for new parchment. Hansen compared the results with a study conducted on leather [38], and made the same general conclusion that the techniques used were not sensitive enough to differentiate between the subtle disparities between samples. A more accurate measure of the mechanical alterations induced from a change in water content could be made by performing shear stress measurements. The shear stress is measured as the component of stress that is coplanar with a material cross section and is used to describe the stress state in which the shape of a material will change without any particular volume change. The shear stress measurement would produce a clearer idea of the size of the stress imposed on a parchment from changing water content at different $\mathrm{RH}$.

Hansen's study recommends that the storage RH should be kept between $30-40 \%$ and bases this recommendation on the finding that there was a large change in mechanical properties of parchment when the $\mathrm{RH}$ was lower than $25 \%$. Hansen recommends $30 \% \mathrm{RH}$ as a lower limit to account for a $+/-5 \%$ fluctuation. The upper limit of $40 \%$ was recommended to prevent microbe growth and was based on the work by Valetin et al. [39]. Valentin reported 'The microorganisms analysed exhibited a considerable retardation of their biological activity in a range of oxygen levels of $0.1-1.1 \%$ and RH lower than 43\%'. The microbe growth rate at $43 \% \mathrm{RH}$ was found to be approximately two thirds that of the $75 \% \mathrm{RH}$ environment and half that of a $100 \% \mathrm{RH}$ environment. Furthermore, the study found that despite this growth reduction, over a period of 3 weeks the biological activity, which indicated the presence of microbes was almost the same in all the $\mathrm{O}_{2}$ environments. Therefore the upper limit recommended by Hansen is not strongly supported by the work of Valentin and should be considered carefully.

Hansen's study had the potential to show how the elasticity of parchments were affected by RH. However, because the samples selected did not account for the large mechanical variation that may occur in orientation 
of collagen fibres within different parts of the animal hide and randomly sampled from parchment artefacts the variance in the data was too large to support strong conclusions. While random sampling is valuable in highlighting variation in a parchment, it means that tensile measurements are more difficult to compare. Moving forwards, a more useful sampling method would have taken all the samples from the same region of the same parchment; this would have reduced the amount of variation between samples and improved the repeatability of the findings.

\section{Conclusions and further work}

There are two common elements of the studies discussed. Firstly, the data shows that there is substantial disparity between samples, thus indicating that consideration needs to be given to the variability of parchments within collections before appropriate storage conditions can be recommended. Secondly, they show that the largest disparity is between new parchments and historic parchments, and in this respect, they provide evidence that the respective quantities of collagen and gelatine in a parchment will be the deciding factor for the most appropriate storage $\mathrm{RH}$.

The studies considered in this review show there is to date no conclusive scientific evidence to suggest a range of RH that is ideal for parchment storage, and an appropriate $\mathrm{RH}$ range cannot be made with certainty. The evidence suggests that an $\mathrm{RH}$ below $30 \%$ will cause a significant increase in internal stress within parchment; there is also evidence that an $\mathrm{RH}$ of approximately $40-45 \%$ reduces the volume change due to water exchange (i.e. the hygric expansion coefficient of the parchment). This is also the $\mathrm{RH}$ region where the water content of gelatine is approximately 14\% [21] and it is near to the point where there is a complete monolayer of water coverage of the gelatine molecular chain [40].

To understand how $\mathrm{RH}$ affects historic parchment artefacts and determine which $\mathrm{RH}$ range is most appropriate for parchment storage we need to consider the following questions:

1. What change in water content is required to induce a significant internal stress that affects the structural integrity of the collagen, and gelatine phases and the interface between them?

2. Is water distribution even throughout the collagen, gelatine and interface phases, or does water have greater affinity toward one phase?

3. Do fluctuations in water content affect the structural integrity of the collagen, interface, and gelatine phases, and if so, are rapid fluctuations of water content more harmful than slower fluctuations?
4. If parchment is stored at a fixed $\mathrm{RH}$, does it reach equilibrium?

Further studies analysing the effects of $\mathrm{RH}$ on parchment artefacts need to take account of the complexity of the interface between collagen and gelatine. Understanding how each structural phase of a parchment object is affected by changes in RH, and how they interact with one another is crucial for determining appropriate environmental conditions for parchment storage. It is also necessary to consider if there is a particular facet of the parchment that, in terms of preservation, is more important. For example, because ink is applied to the surfaces of parchments, and penetrates inwards; a majority of the ink will more likely be contained in the surface gelatine phase of the parchment. Since the collagen phase will be able to resist water changes at low temperatures [21,41], should we consider the interface and gelatine phases to be structurally more important, and focus research to determine which $\mathrm{RH}$ conditions best preserve these structural phases?

Furthermore, it is important to consider which samples are most appropriate to model changes in water content. For instance, [36] found that artificially aged parchments were not comparable to historic parchments. They report no correlation with regard to the amount of water taken up by the historical and artificially aged samples. This indicates that the mechanism of degradation of collagen is an important factor that needs to be considered when designing future studies, and artificially aged parchments may not always provide an adequate model for the behaviour of historical parchments at different RH.

The most useful information obtained from the studies reviewed is that appropriate environmental $\mathrm{RH}$ conditions for the preservation of parchment artefacts are different for new and historical parchments. This is most likely due to differences in the thickness and relative proportions of collagen to gelatine in a parchment artefact. It is has been assumed in the studies evaluated in this review, that the proportion of collagen to gelatine is always greater in new parchment than in historical parchment. Although it is reasonable to assume that historical parchment will contain considerable proportions of gelatine, it is also possible that due to the manufacturing procedure, new parchment will also contain large quantities of gelatine, and this needs to be accounted for when selecting samples.

The research evaluated has shown that the understanding of the effects of changes in $\mathrm{RH}$ on parchment structure is still limited. There remains a lack of clarity as to the most suitable parameters to measure when determining how parchment artefacts are affected by a change in $\mathrm{RH}$ and subsequently water content. Furthermore, there is no single analytical technique that will 
provide all the answers and complementary analysis and interpretation from several techniques will be required.

Overall, the studies reviewed have shown that controlling $\mathrm{RH}$ to approximately $40 \%$ can reduce hygroscopic expansion of the parchment structure. They have also shown that large internal stresses occur when the $\mathrm{RH}$ is lower then $30 \%$, and this can result in undesirable changes of the mechanical and physical properties of a parchment. These results provide an appropriate starting point for future studies.

\section{The importance of this review for parchment collection management}

This review has brought together experimental findings from several publications on the effects of $\mathrm{RH}$ on parchment. One of the most central features of the studies is the fact several different aspects of the parchment have been assessed and found to be affected by changes in $\mathrm{RH}$. For example, Weiner et al. recognised the role high $\mathrm{RH}$ plays in the conversion of perturbed collagen into gelatine, Schilling et al. and Dernovšková et al. observed the change in volume of parchment from water absorption and desorption at different $\mathrm{RH}$, and Hanson et al. recognised the change in mechanical properties of parchment with changes in $\mathrm{RH}$. This is important when considering appropriate storage conditions because it highlights that there are different facets within parchment that change with $\mathrm{RH}$ changes. Moreover, knowing and understanding these changes allows collection managers to make more informed choices when considering $\mathrm{RH}$ within their collections.

On a more practical level this review discusses the interaction of water with parchment and this is useful in the context of parchment conservation. For example, in terms of the upper limit of $65 \% \mathrm{RH}$ recommended by Weiner et al., this is already in the conscious of collection managers because of the threat of mould growth at elevated RH levels. However, with regard to conservation treatments it does bring forward the debate as to whether humidification above a certain level - which would allow perturbed collagen to unfold into gelatine is appropriate to remove unwanted creases and folds. Likewise, exposing parchment to low $\mathrm{RH}$ causes a loss of elasticity and it might be useful to prevent the $\mathrm{RH}$ in reading rooms dropping below a certain value.

\section{Competing interests}

The authors declare they have no competing interests.

\section{Authors' contributions}

The work carried out in preparation of this paper was performed equally between LGG and TJW. Both authors read and approved the final manuscript.

\section{Acknowledgements}

The authors would like to thank Nancy Bell of The National Archives for her valuable contribution to this work, and the Science and Heritage Programme for financial support.

Received: 20 February 2013 Accepted: 4 April 2013 Published: 24 April 2013

\section{References}

1. Gonzalez LG, Wade M, Bell N, Thomas K, Wess TJ: Using attenuated total reflection- Fourier transform infrared spectroscopic (ATR-FTIR) to study the molecular conformation of parchment artifacts in different macroscopic states. App/ Spectrosc 2013, 67:158-162.

2. Kennedy CJ, Hiller JC, Lammie D, et al: Microfocus X-ray diffraction of historical parchment reveals variations in structural features through parchment cross sections. Nano Letters 2004, 4:1373-1380.

3. Gonzalez LG, Wess TJ: Use of attenuated total reflectance - Fourier transform infrared spectroscopy to measure collagen degradation in historical parchments. App/ Spectrosc 2008, 62:1108-1114.

4. Sionkowska A: Thermal denaturation of UV-irradiated wet rat tail tendon collagen. Int J Biol Macromol 2005, 35:145-149.

5. Nielsen K: Visual Damage Assessment. In Improved damage assessment of parchment. Edited by Larsen R. Brussels: Office of official publications of the European Communities; 2007.

6. Ghioni C, Hiller JC, Kennedy CJ, et al: Evidence of a distinct lipid fraction in historical parchments: a potential role in degradation? Journal of Lipid Research 2005, 46:2726-2734

7. Možir A, Gonzalez LG, Cigić IK, et al: A study of degradation of historic parchment using small-angle X-ray scattering, synchrotron-IR and multivariate data analysis. Anal Bioanal Chem 2012, 402:1559-1566.

8. Wess TJ: Collagen Fibrillar Structure and Hierarchies. In Collagen: Structure and Mechanics. Edited by Fratzl P. New York: Springer; 2008.

9. Ramachandran GX, Chandrasekharan R: Interchain hydrogen bonds via bound water molecules in the collagen triple helix. Biopolymers 1968, 6:1649-1658.

10. Pineri $\mathrm{MH}$, Escoubes $\mathrm{M}$, Roche $\mathrm{G}$ : Water-collagen interactions: Calorimetric and mechanical experiments. Biopolymers 1978, 17:2799-2815.

11. Zhang D, Chippada U, Jordan K: Effect of the structural water on the mechanical properties of collagen-like microfibrils: a molecular dynamics study. Ann Biomed Eng 2007, 35:1216-1230.

12. Mrevlishvilli GM, Khutsisvilli VG, Monaselidze DP, Dzhaparidze GS: Hydration of native-type collagen fibers. Dokl Akad Nauk SSSR 1974, 215:457-459.

13. Mrevlishvilli GM: Low-temperature heat capacity of biomacromolecules and the entropic cost of bound water in proteins and nucleic acids. Thermochim Acta 1998, 308:49-54.

14. Mrevlishvilli GM, Svintradze DV: Complex between triple helix of collagen and double helix in DNA in aqueous solution. Int J Biol Macromol 2005, 35:243-245.

15. Leikina E, Mertts MV, Kuznetsova N, Leikin S: Type I collagen is thermally unstable at body temperature. Proceedings of the National Academy of Science 2002, 99:1314-1318.

16. Galant S: Formation of collagen-like structure of gel after radiation modification of gelatin. Colloid \& Polymer Science 1983, 261:445-451.

17. Varani J, Dame MK, Rittie L, et al: Decreased collagen production in chronologically aged skin. Roles of Age-dependent alteration in fibroblast function and defective mechanical stimulation. American Journal of Pathology 2006, 168:1861-1868.

18. Budrugeac $P$, Miu L: Effect of accelerated thermal ageing on the therma behaviour of the recently made parchments. J Therm Anal Calorim 2008, 94:335-342.

19. Chou DH, Morr C: Protein-water interactions and functional properties. Journal of the American Oil Chemists' Society 1979, 56:53-62.

20. Bradbury EM, Burge RE, Randallan JT, Wilkinson DGR: The polypeptide chain configurations of native and denatured collagen fibres. Discuss Faraday Society 1958, 25:173-185.

21. Yakimets I, Wellner N, Smith AC, et al: Mechanical properties with respect to water content of gelatin films in glassy state. Polymer 2005, 46:12577-12585.

22. Shamblin SL, Hancock BC, Zografi G: Water vapor sorption by peptides, proteins and their formulations. Eur J Pharm Biopharm 1998, 45:239-247. 
23. Bull HB: Absorption of water vapor by proteins. J Am Chem Soc 1944, 66:1499-1507.

24. Schreiber R, Gareis H: Gelatin Handbook. Wienheim: Theory and Industrial practise. Wiley -VCH Verlag - $\mathrm{GmbH}$ and $\mathrm{Co} ; 2007$.

25. Baier RE: Applied Chemistry at Protein Interfaces. Washington DC: American Chemical Society; 1975

26. Okamoto Y, Saeki K: Phase transition of collagen and gelatin. Colloid \& Polymer Science 1964, 194:124-135.

27. de Groot J: Damage assessment of collagen in historical parchment with microscopy techniques. PhD Thesis. London: University of London, Birkbeck College, Department of Biological Sciences; 2007.

28. Aliev AE: Solid state NMR studies of collagen based parchments and gelatin. Biopolymers 2005, 77:230-245.

29. Petushkova YP, Nikolaev GM: Nuclear Magnetic Resonance Study of Parchment and Leather. Restaurator 1983, 5:242-248.

30. Thompson G: The Museum Environment. London: Butterworths; 1986.

31. Block SS: Humidity Requirements for Mold Growth. Appl Environ Microbiol 1953, 7:287-293.

32. Weiner S, Kustanovich Z, Gil-Av E, Traub W: Dead Sea Scroll parchments: unfolding of the collagen molecules and racemization of aspartic acid. Nature 1980, 287:820-823.

33. Gustavson KH: Chemistry and Reactivity of Collagen. New York: Academic Press Inc; 1956.

34. Schilling MR, Ginell WS: The Effects of Relative Humidity Changes on Dead Sea Scrolls Parchment Samples. In Preprints of the 10th Triennial Meeting of ICOM Committee for Conservation. Washington D.C, USA:; 1993:50-56

35. Dernovskova J, Jirsova H, Zelinger J: An investigation of the Hygroscopicity of Parchment subjected to different treatments. Restaurator 1995, 16:31-44.

36. Bowden D, Brimblecombe P: Thermal Response of Parchment and Leather to Relative Humidity Changes. In Microanalysis of Parchment. Edited by Larsen R. London: Archetype Publications Ltd; 2002.

37. Hansen EF, Lee SN, Sobel H: The effects of relative humidity on some physical properties of modern vellum. Implications for the Optimum Relative Humidity for the Display and Storage of Parchment. J Am Inst Conserv 1991, 31:325-342.

38. Evans WD, Critchfield CL: The effects of atmospheric moisture on the physical properties of vegetable and chrome tanned calf leathers. Journal of Research of the National Bureau of Standards 1933, 11:147.

39. Valentin N, Lindstorm M, Preusser F: Microbial control by low oxygen and low relative humidity environment. Stud Conserv 1990, 35:222-230.

40. Vackier M-C, Hills BP, Rutledge DN: An NMR relaxation study of the state of water in gelatin gels. J Magn Reson 1999, 138:36-42.

41. Gevorkian SG, Allahverdyan AE, Gevorgyan DS, Simonian A: Thermal (in) stability of type I collagen fibrils. Phys Rev Lett 2009, 102:48101-48104.

doi:10.1186/2050-7445-1-14

Cite this article as: Gonzalez and Wess: The effects of hydration on the collagen and gelatine phases within parchment artefacts. Heritage Science 2013 1:14.

Publish with ChemistryCentral and every
scientist can read your work free of charge
"Open access provides opportunities to our
colleagues in other parts of the globe, by allowing
anyone to view the content free of charge."
W. Jeffery Hurst, The Hershey Company.
- available free of charge to the entire scientific community
- peer reviewed and published immediately upon acceptance
- cited in PubMed and archived on PubMed Central
- yours - you keep the copyright
Submit your manuscript here:
htpp://www.chemistrycentral.com/manuscript/

